\title{
Efficient covering designs of the complete graph
}

\author{
Yair Caro * \\ and \\ Raphael Yuster ${ }^{\dagger}$ \\ Department of Mathematics \\ University of Haifa-ORANIM, Tivon 36006, Israel. \\ AMS Subject Classification: 05B05,05B40 (primary), \\ 05B30,51E05,94C30,62K05,62K10 (secondary).
}

Submitted: November 1, 1996; Accepted: February 3, 1997

\begin{abstract}
Let $H$ be a graph. We show that there exists $n_{0}=n_{0}(H)$ such that for every $n \geq n_{0}$, there is a covering of the edges of $K_{n}$ with copies of $H$ where every edge is covered at most twice and any two copies intersect in at most one edge. Furthermore, the covering we obtain is asymptotically optimal.
\end{abstract}

\section{Introduction}

All graphs considered here are finite, undirected and simple, unless otherwise noted. For the standard graph-theoretic notations the reader is referred to [5]. Let $H=\left(V_{H}, E_{H}\right)$ be a graph. An $H$-covering design of a graph $G=\left(V_{G}, E_{G}\right)$ is a set $L=\left\{G_{1}, \ldots G_{s}\right\}$ of subgraphs of $G$ such that each $G_{i}$ is isomorphic to $H$ and every edge $e \in E_{G}$ appears in at least one member of $L$. The $H$-covering number of $G$, denoted by $\operatorname{cov}(G, H)$, is the minimum number of members in an $H$-covering design of $G$. (If there is an edge of $G$ which cannot be covered by a copy of $H$, we put $\operatorname{cov}(G, H)=\infty)$. Clearly, $\operatorname{cov}(G, H) \geq\left|E_{G}\right| /\left|E_{H}\right|$. In case equality holds, the $H$-covering design is called an $H$-decomposition (or $H$-design) of $G$. Two trivial necessary conditions for a decomposition are that $\left|E_{H}\right|$ divides $\left|E_{G}\right|$ and that $\operatorname{gcd}(H)$ divides $\operatorname{gcd}(G)$ where the $\operatorname{gcd}$ of a graph

*e-mail: zeac603@uvm.haifa.ac.il

${ }^{\dagger}$ e-mail: raphy@math.tau.ac.il 
THE ELECTRONiC JouRnal of COMBinatorics 4 (1997), \#R10

is the greatest common divisor of the degrees of all the vertices. In case $G=K_{n}$, it was shown by Wilson in [17] that the two necessary conditions are also sufficient, provided $n \geq n_{0}(H)$, where $n_{0}(H)$ is a sufficiently large constant. If, however, the necessary conditions do not hold, the best one could hope for is an $H$-covering design of $K_{n}$ where the following three properties hold:

1. 2-overlap: Every edge is covered at most twice.

2. 1-intersection: Any two copies of $H$ intersect in at most one edge.

3. Efficiency: $s\left|E_{H}\right|<\left(\begin{array}{l}n \\ 2\end{array}\right)+c(H) \cdot n$, where $s$ is the number of members in the covering, and $c(H)$ is some constant depending only on $H$.

The papers of Mills and Mullin [12] and of Brouwer [4], provide an excellent survey of covering designs. Covering designs with the 2-overlap property were first introduced in statistical designs by [10] and are also mentioned in [2], [6] and [11], Covering designs with the 1-intersection property (also called super-simple designs) are mentioned by Adams et. al. in [1], Teirlinck [15, 16], Fort and Hedlund [8], Brouwer [3] and Schreiber [14]. The existence of efficient Covering designs of complete hypergraphs was first proved by Rödl in [13].

Our main result is that $H$-covering designs of $K_{n}$, having these three properties, exist for every fixed graph $H$, and for all $n \geq n_{0}(H)$ :

Theorem 1.1 Let $H$ be a fixed graph. There exists $n_{0}=n_{0}(H)$ such that if $n \geq n_{0}, K_{n}$ has an $H$-covering design with the 2-overlap, 1-intersection, and efficiency properties.

\section{Proof of the main result}

We shall prove Theorem 1.1 whenever $H=K_{h}$ is a complete graph. This suffices, since if $H$ is not a complete graph, it is known by Wilson's theorem [17] that there exists an $h_{0}=h_{0}(H)$ such that $K_{h_{0}}$ has an $H$-decomposition. By applying Theorem 1.1 to $K_{h_{0}}$, we shall obtain an $n_{0}=n_{0}\left(h_{0}\right)=n_{0}(H)$, such that if $n \geq n_{0}, K_{n}$ has a $K_{h_{0}}$-covering design with the 2-overlap and 1-intersection properties and such that $\left(\begin{array}{c}h_{0} \\ 2\end{array}\right) s<\left(\begin{array}{c}n \\ 2\end{array}\right)+h_{0}^{3} \cdot n$, where $s$ is the number of members in the covering. Thus, there is an $H$-covering design of $K_{n}$ with the 2-overlap and 1-intersection properties, and with $s \frac{\left(\begin{array}{c}h_{0} \\ 2\end{array}\right)}{\left|E_{H}\right|}$ elements, such that $s \frac{\left(\begin{array}{c}h_{0} \\ 2\end{array}\right)}{\left|E_{H}\right|}\left|E_{H}\right|<\left(\begin{array}{c}n \\ 2\end{array}\right)+h_{0}^{3} \cdot n=\left(\begin{array}{c}n \\ 2\end{array}\right)+c(H) \cdot n$.

Fix $K_{h}$, where $h \geq 3$ (for $h=2$ the result is trivial), and let $h_{1}$ be the minimum positive integer such that whenever $n \geq h_{1}$ and $\left(\begin{array}{c}h \\ 2\end{array}\right)$ divides $\left(\begin{array}{l}n \\ 2\end{array}\right)$, and $h-1$ divides $n-1, K_{n}$ has a $K_{h^{-}}$ decomposition. As mentioned before, the existence of $h_{1}$ is guaranteed by Wilson's Theorem [17].

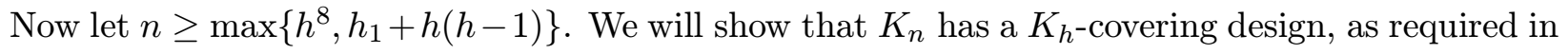


THE ElECTRONIC JOURNAL OF COMBINATORICS 4 (1997), \#R10

Theorem 1.1. Let $k$ be the minimum positive integer such that $\left(\begin{array}{c}h \\ 2\end{array}\right)$ divides $\left(\begin{array}{c}n-k \\ 2\end{array}\right)$ and $h-1$ divides $n-k-1$. It is easy to see that $0 \leq k<h(h-1)$. If $k=0$ we are done, since in this case $n$ satisfies the conditions in Wilson's Theorem, and there is a $K_{h}$-decomposition of $K_{n}$. Assume, therefore, that $1 \leq k<h(h-1)$, and put $r=n-k$. Note that $r>h_{1}$. Partition the vertices of $K_{n}$ into two subsets. The big subset has $r$ vertices, namely $B=\left\{a_{1}, \ldots, a_{r}\right\}$. The small subset has $k$ vertices, namely $S=\left\{b_{1}, \ldots, b_{k}\right\}$. We create the members of our efficient covering design in three stages.

Stage 1: Let $B_{0}$ be the subgraph induced by the vertices $\left\{a_{1}, \ldots, a_{r-1}\right\}$. Note that $B_{0}$ is a complete graph on $r-1$ vertices, and since $h-1$ divides $r-1$, there exists a $K_{h-1}$-factor in $B_{0}$. (Recall that an $X$-factor of a graph is a set of vertex-disjoint copies of $X$ which cover all the vertices of the graph). Let $F_{1}$ be such a factor. We repeat the following process for $i=2, \ldots, k$. Let $B_{i-1}$ be the graph obtained from $B_{i-2}$ after the edges of the members of $F_{i-1}$ have been removed. Let $F_{i}$ be a $K_{h-1}$-factor in $B_{i-1}$. In order to show that our process works, we need to show that a $K_{h-1}$-factor exists in $B_{i-1}$. We prove this by induction on $i$. For $i=1$, this is simply the factor $F_{1}$ defined above. Assume the claim holds for all $j<i$. This implies that $B_{i-1}$ is regular of degree $(r-2)-(i-1)(h-2)$. According to the theorem of Hajnal and Szemerédi [9] if $(r-2)-(i-1)(h-2) \geq \frac{h-2}{h-1}(r-1)$ then $B_{i-1}$ has a $K_{h-1}$-factor. Indeed,

$$
(r-2)-(i-1)(h-2) \geq(r-2)-(k-1)(h-2)>(r-2)-h(h-1)(h-2)>r-h^{3} .
$$

Since $r-\frac{r-1}{h-1}>\frac{h-2}{h-1}(r-1)$ it suffices to show that $r-h^{3} \geq r-\frac{r-1}{h-1}$ and this holds since $r=n-k>h^{4}$. Having defined the $K_{h-1}$-factors $F_{1}, \ldots, F_{k}$, we now define a set $L_{1}$ of edge-disjoint copies of $K_{h}$ in our $K_{n}$, which cover all the edges between $S$ and $\left\{a_{1}, \ldots, a_{r-1}\right\}$. This is done by joining the vertex $b_{i}$ to every member of $F_{i}$, for $i=1, \ldots, k$. Note that whenever we join $b_{i}$ to a member of $F_{i}$ we obtain a copy of $K_{h}$. Note also that $L_{1}$ has exactly $k(r-1) /(h-1)$ members.

Stage 2: Since $r \geq h_{1}$, and since $h-1$ divides $r-1$ and $\left(\begin{array}{l}h \\ 2\end{array}\right)$ divides $\left(\begin{array}{l}r \\ 2\end{array}\right)$, we have by Wilson's Theorem that the subgraph induced by $B$ (which is a $K_{r}$ ), has a $K_{h}$-decomposition. Fix a labeled $K_{h}$-decomposition $D$ of this $K_{r}$. That is, $D$ is a set of $\left(\begin{array}{c}r \\ 2\end{array}\right) /\left(\begin{array}{c}h \\ 2\end{array}\right) h$-subsets of $\left\{a_{1}, \ldots, a_{r}\right\}$, where for each $1 \leq i<j \leq r$, the pair $\left(a_{i}, a_{j}\right)$ appears in exactly one member of $D$. If $\pi$ is any permutation of $\{1, \ldots, r\}$ then let $D_{\pi}$ be the labeled $K_{h}$-decomposition obtained from $D$ by replacing each appearance of $a_{i}$ in any member of $D$ with $\pi\left(a_{i}\right)$, for $i=1, \ldots, r$. Our aim is to show that there exists a permutation $\pi$, and a set $L^{*}$ of less than $h^{5}$ members of $L_{1}$ (recall that $L_{1}$ is constructed in stage 1), such that every member of $D_{\pi}$ intersects every member of $L_{1} \backslash L^{*}$ in at most one edge. In order to achieve this goal, we pick $\pi$ randomly, where each of the $r$ ! permutations is equally likely. Consider two distinct edges $\left(a_{i}, a_{j}\right)$ and $\left(a_{k}, a_{l}\right)$ which both appear in the same member of $L_{1}$ (note that when $h=3$, there is no such pair, since every member of $L_{1}$ contains only two vertices of $B$ ). We call such a pair of edges $D_{\pi}$-bad if they both appear in the same member of $D_{\pi}$. We shall 
THE ELECTRONiC JouRnal of COMBinatorics 4 (1997), \#R10

compute the probability that two fixed edges $\left(a_{i}, a_{j}\right)$ and $\left(a_{k}, a_{l}\right)$ are $D_{\pi}$-bad. Consider first the case where $\left(a_{i}, a_{j}\right)$ and $\left(a_{k}, a_{l}\right)$ share an endpoint, say $a_{k}=a_{i}$. Since $\pi$ is random, the probability that $\left(a_{i}, a_{j}\right)$ and $\left(a_{i}, a_{l}\right)$ appear in the same member of $D_{\pi}$ is exactly $\frac{h-2}{r-2}$. To see this, fix $\pi\left(a_{i}\right)$ and $\pi\left(a_{j}\right)$, and let $Q$ denote the unique member of $D$ which contains both $\pi\left(a_{i}\right)$ and $\pi\left(a_{j}\right)$. There are $r-2$ possible choices for $\pi\left(a_{l}\right)$, where $h-2$ of them result in a member of $Q$. Thus, $D_{\pi}$ is bad with probability $\frac{h-2}{r-2}$, given that $\pi\left(a_{i}\right)$ and $\pi\left(a_{j}\right)$ are known. Note, however, that the expression $\frac{h-2}{r-2}$ does not depend on the specific choices for $\pi\left(a_{i}\right)$ and $\pi\left(a_{j}\right)$. Now consider the case where $\left(a_{i}, a_{j}\right)$ and $\left(a_{k}, a_{l}\right)$ are two independent edges (this is possible only if $h-1 \geq 4$, since every member of $L_{1}$ contains only $h-1$ vertices from $B$ ). By a similar reasoning to the above, the probability that both these edges appear in the same member of $D_{\pi}$ is exactly $\frac{h-2}{r-2} \frac{h-3}{r-3}$. There are $(h-1)(h-2)(h-3) / 2$ pairs of adjacent edges of the form $\left(a_{i}, a_{j}\right),\left(a_{i}, a_{l}\right)$ in every member of $L_{1}$. Thus, there are $k \frac{r-1}{h-1}(h-1)(h-2)(h-3) / 2$ such pairs in all the members of $L_{1}$. There are $3\left(\begin{array}{c}h-1 \\ 4\end{array}\right)$ pairs of two independent edges of the form $\left(a_{i}, a_{j}\right),\left(a_{k}, a_{l}\right)$ in every member of $L_{1}$. Thus there are $3 k \frac{r-1}{h-1}\left(\begin{array}{c}h-1 \\ 4\end{array}\right)$ such pairs in all the members of $L_{1}$. Therefore, if $\mu$ is the expected number of $D_{\pi}$-bad pairs, then

$$
\begin{gathered}
\mu=k \frac{r-1}{h-1} \frac{(h-1)(h-2)(h-3)}{2} \frac{h-2}{r-2}+k \frac{r-1}{h-1} 3\left(\begin{array}{c}
h-1 \\
4
\end{array}\right) \frac{h-2}{r-2} \frac{h-3}{r-3}< \\
\frac{h^{5}}{2}+\frac{3}{24} h^{7} \frac{r-1}{(r-2)(r-3)}<h^{5} .
\end{gathered}
$$

Thus, there exists a permutation $\pi$ such that the number of $D_{\pi}$-bad pairs is less than $h^{5}$. Fix such a permutation, and let $L_{2}=D_{\pi}$. Let $L^{*}$ be the set of all members of $L_{1}$ which contain a $D_{\pi}$-bad pair. Clearly, $\left|L^{*}\right|<h^{5}$. Thus, every member of $L_{2}$ intersects every member of $L_{1} \backslash L^{*}$ in at most one edge. Put $L_{3}=L_{2} \cup\left(L_{1} \backslash L^{*}\right)$.

Stage 3: Every edge of $K_{n}$ appears in at most two members of $L_{3}$ and any two members of $L_{3}$ intersect in at most one edge. However, there may still be uncovered edges. In fact, all the $\left(\begin{array}{l}k \\ 2\end{array}\right)$ edges connecting two members of $S$ are not covered, and all the $k$ edges of the form $\left(b_{i}, a_{r}\right)$, for $i=1, \ldots, k$, are not covered. Furthermore, each member of $L^{*}$ covers $h-1$ edges connecting some $b_{i} \in S$ to a subset of $h-1$ vertices of $\left\{a_{1}, \ldots, a_{r-1}\right\}$, and these edges are uncovered in $L_{3}$. Thus there are $\left|L^{*}\right|(h-1)$ uncovered edges of this form. Hence, if $M$ denotes the set of uncovered edges, we have that

$$
|M|=\left(\begin{array}{l}
k \\
2
\end{array}\right)+k+\left|L^{*}\right|(h-1)<h^{6} .
$$

The crucial point is that the number of uncovered edges is bounded by a constant depending only on $h$. We shall show how to sequentially create a set $L_{4}$ of copies of $K_{h}$, beginning with $L_{4}=\emptyset$, 
THE ELECTRONiC JouRnal of COMBinatorics 4 (1997), \#R10

where at each stage, a new copy of $K_{h}$ containing at least one non-covered edge by members of $L_{3} \cup L_{4}$, is added to $L_{4}$ (thus $\left|L_{4}\right|<h^{6}$ ) and such that the following three invariants are maintained:

1. Every edge is covered at most twice by members of $L_{3} \cup L_{4}$.

2. Any two members of $L_{3} \cup L_{4}$ intersect in at most one edge.

3. If $L_{4}$ already contains $j$ members, then any vertex of $B \cup S$ is adjacent to at most $j h+h^{3}$ edges which are covered twice by members of $L_{3} \cup L_{4}$.

Note that at the beginning of the process, when $L_{4}=\emptyset$, the first two invariants hold, since they hold for $L_{3}$. We must show that the third invariant holds initially, when $j=0$. Indeed, in $L_{3}$, all the edges adjacent to a vertex of $S$ are either non-covered, or covered once in $L_{1}$. Now consider a vertex $a_{i} \in B$. If $i<r, a_{i}$ is adjacent to exactly $(h-2) k$ edges which are covered twice by members of $L_{1} \cup L_{2}$ (recall that $a_{r}$ is not adjacent to any edge which is covered in $L_{1}$ ). Since $L_{3} \subset L_{1} \cup L_{2}$, we have that any vertex in $B \cup S$ is adjacent to at most $(h-2) k<h^{3}$ edges which are covered twice by members of $L_{3}$.

Suppose $L_{4}$ already contains $j$ members, and there still exists an uncovered edge $e=\left(q_{1}, q_{2}\right)$ in $M$. We shall find a set $Q=\left\{q_{3}, \ldots, q_{h}\right\}$ of $h-2$ vertices in $B \cup S$, and add the complete graph $K_{h}$ induced by $\left\{q_{1}, q_{2}, \ldots, q_{h}\right\}$ to $L_{4}$, while maintaining our three invariants. We select the elements of $Q$ sequentially. The first element, $q_{3}$, needs to have the property that $\left(q_{1}, q_{3}\right)$ is not covered twice, and $\left(q_{2}, q_{3}\right)$ is not covered twice. Indeed there are at most $2\left(j h+h^{3}\right)$ vertices of $(B \cup S) \backslash\left\{q_{1}, q_{2}\right\}$ which are ruled out as candidates for $q_{3}$. Since

$$
2\left(j h+h^{3}\right)<2\left(h^{7}+h^{3}\right) \leq h^{8}-2 \leq n-2
$$

we can find the desired $q_{3}$. It is important to note that there does not exist any member of $L_{3} \cup L_{4}$ which contains both $\left(q_{1}, q_{3}\right)$ and $\left(q_{2}, q_{3}\right)$, since this would require it to contain $\left(q_{1}, q_{2}\right)$ which we assume to be uncovered. Therefore, invariants 1 and 2 till hold. Suppose we have already found appropriate vertices $q_{3}, \ldots, q_{i}$, where $i<h$, and we wish to find $q_{i+1}$. Our requirements of $q_{i+1}$ are as follows: All the edges $\left(q_{t}, q_{i+1}\right)$ for $t=1, \ldots, i$ should each be covered at most once, and for each once-covered edge $\left(q_{t}, q_{p}\right)$ where $1 \leq t<p \leq i, q_{i+1}$ does not appear in the unique copy of $L_{3} \cup L_{4}$ containing $\left(q_{t}, q_{p}\right)$. These requirements rule out at most

$$
i \cdot\left(j h+h^{3}\right)+\left(\begin{array}{l}
i \\
2
\end{array}\right)(h-2)
$$

possible candidates for $q_{i+1}$ from $(B \cup S) \backslash\left\{q_{1}, \ldots, q_{i}\right\}$. In order to show that $q_{i+1}$ can be selected we need to show that

$$
n-i>i\left(j h+h^{3}\right)+\left(\begin{array}{l}
i \\
2
\end{array}\right)(h-2) .
$$


Indeed,

$$
i\left(j h+h^{3}\right)+\left(\begin{array}{l}
i \\
2
\end{array}\right)(h-2) \leq(h-1)\left(h^{7}+h^{3}\right)+\left(\begin{array}{c}
h-1 \\
2
\end{array}\right)(h-2)<h^{8}-(h-1) \leq n-i .
$$

Our construction of $Q$ shows that after adding the $K_{h}$ subgraph induced by $\left\{q_{1}, \ldots, q_{h}\right\}$ as the $j+1$ 'th element to $L_{4}$, invariants 1 and 2 still hold. Note also that invariant 3 holds as any vertex may only have at most $h-1$ edges which are now covered twice, and which were not covered twice prior to this stage. (The only vertices for which this may happen are $q_{1}, \ldots, q_{h}$ ).

In order to complete our proof we only need to show that if $L=L_{3} \cup L_{4}$ contains $s$ elements then $s\left(\begin{array}{l}h \\ 2\end{array}\right)<\left(\begin{array}{l}n \\ 2\end{array}\right)+h^{3} n$. Clearly, it suffices to show that

$$
\operatorname{sh}(h-1)<n(n-1)+h^{3}(n-1) \cdot \psi
$$

$L_{4}$ contains less than $h^{6}$ members. $L_{1}$ contains exactly $k(r-1) /(h-1)$ members, and $L_{2}$ contains exactly $\left(\begin{array}{l}r \\ 2\end{array}\right) /\left(\begin{array}{l}h \\ 2\end{array}\right)$ members. Thus,

$$
s<h^{6}+k \frac{r-1}{h-1}+\frac{\left(\begin{array}{c}
r \\
2
\end{array}\right)}{\left(\begin{array}{c}
h \\
2
\end{array}\right)} \cdot \psi
$$

We shall prove (1) using (2) and using the facts that $k<h(h-1), r=n-k$ and $n \geq h^{8}$. Indeed

$$
\begin{gathered}
\operatorname{sh}(h-1)<h^{7}(h-1)+h k(r-1)+r(r-1)=h^{8}-h^{7}+h k n-h k^{2}-h k+n^{2}-2 k n+k^{2}-n+k< \\
h^{8}-h^{3}+h k n+n^{2}-2 k n-n<n(n-1)+h^{3}(n-1) .
\end{gathered}
$$

\section{3- Concluding remarks and an open problem}

When $H=K_{h}$, the constant $n_{0}(H)$ in Theorem 1.1 is shown in the proof to be no larger than $\max \left\{h^{8}, h_{1}+h(h-1)\right\}$, where $h_{1}=h_{1}(h)$ is the corresponding constant in Wilson's Theorem. However, the best known bound for $h_{1}$ (and, consequently, for $n_{0}(H)$ ), is rather large, and highly exponential in $h[7]$. It is plausible, however, that the statement of Theorem 1.1 is still valid for $n_{0}(H)$ which is much smaller. In fact, we conjecture the following:

Conjecture 3.1 There exists a positive constant $C$ such that for all $h \geq 2$, if $n \geq C h^{2}$ then $K_{n}$ has a $K_{h}$ covering design where each edge is covered at most twice and any two copies intersect in at most one edge. 
THE ELECTRONiC JouRnal of COMBinatorics 4 (1997), \#R10

Note that a positive answer to Conjecture 3.1 requires a proof which does not use Wilson's Theorem, as improving Wilson's constant to $O\left(h^{2}\right)$ is unlikely. The $h^{2}$ factor in Conjecture 3.1 cannot be reduced since we have the following simple $0.25 h^{2}$ lower bound: Assume that $h \geq 10$. If $n=\left\lfloor 0.25 h^{2}\right\rfloor$ then any $K_{h}$-covering of $K_{n}$ contains $\left(\begin{array}{l}n \\ 2\end{array}\right) /\left(\begin{array}{l}h \\ 2\end{array}\right)>h / 2$ members. However, the union of $t K_{h}$-subgraphs with the 1 -intersection property contains at least $h+(h-2)+\ldots+(h-2 t+2)$ vertices. For $t=\lceil h / 2\rceil$ this sum is greater than $0.25 h^{2} \geq n$. Thus, any $K_{h}$-covering of $K_{n}$ does not have the 1-intersection property.

\section{Acknowledgment}

The authors wish to thank T. Etzion and A. Rosa for useful discussions.

\section{References}

[1] P. Adams, E. Bryant and A. Khodkar, On the existence of super-simple designs with block-size 4, Aequationes Mathematicae 51 (1996), 230-246.

[2] N. Alon, Y. Caro and R. Yuster, Covering the edges of a graph by a prescribed tree with minimum overlap, submitted.

[3] A. E. Brouwer, On the packing of quadruples without common triples, Ars Combinatoria 5 (1978), 3-6.

[4] A. E. Brouwer, Block Designs, in: Chapter 14 in "Handbook of Combinatorics", R. Graham, M. Grötschel and L. Lovász Eds. Elsevier, 1995.

[5] J. A. Bondy and U. S. R. Murty, Graph Theory with Applications, Macmillan Press, London, 1976.

[6] Y. Caro, Y. Roditty and Y. Schonheim, Covering the edges of the complete graph with minimum overlap, Manuscript.

[7] Y. Chang, A bound for Wilson's Theorem - III, J. Combinatorial Designs 4 (1996), 83-93.

[8] M. K. Fort and G. A. Hedlund, Minimal covering of pairs by triples, Pacific J. Math. 8 (1958), 709-719. 
THE EleCtronic Journal of COMBinAtorics 4 (1997), \#R10

[9] A. Hajnal and E. Szemerédi, Proof of a conjecture of Erdös, in: Combinatorial Theory and its Applications, Vol. II (P. Erdös, A. Renyi and V. T. Sós eds.), Colloq. Math. Soc. J. Bolyai 4, North Holland, Amsterdam 1970, 601-623.

[10] J. A. John and T. J. Mitchell, Optimal incomplete block designs J. Roy. Statist. Soc. 39B (1977) $39-43$.

[11] D. L. Kreher, G. F. Royle and W. D. Wallis, A family of resolvable regular graph designs, Discrete Math. 156 (1996), 269-275.

[12] W. H. Mills and R. C. Mullin, Coverings and packings, in: Contemporary Design Theory: A collection of Surveys, 371-399, edited by J. H. Dinitz and D. R. Stinson. Wiley, 1992.

[13] V. Rödl, On a packing and covering problem, Europ. J. of Combin. 6 (1985), 69-78.

[14] S. Schreiber, Covering all triples on $n$ marks by disjoint Steiner systems, J. Combin. Theory, Ser. A 15 (1973), 347-350.

[15] L. Teirlinck, On making two Steiner triple systems disjoint, J. Combin. Theory, Ser. A 23 (1977), 349-350.

[16] L. Teirlinck, On large sets of disjoint quadruple systems, Ars Combinatoria 17 (1984), 173-176.

[17] R. M. Wilson, Decomposition of complete graphs into subgraphs isomorphic to a given graph, Congressus Numerantium XV (1975), 647-659. 